\title{
Polarimetry - Scope on the 3.6-m Devasthal Optical Telescope
}

\author{
Umesh Chandra Joshi*, Shashikiran Ganesh, Kiran Singh Baliyan
}

Physical Research Laboratory, Ahmedababd, INDIA

\begin{abstract}
Polarization measurements are very helpful to understand the nature of some of the stellar and extragalactic sources. Light from astronomical objects is in general polarized to some degree and its measurement gives additional information related to the magnetic field, the distribution of scattering material, the non-thermal nature of light, etc. Since the degree of polarization in the majority of astronomical sources is $1-5 \%$, and polarimetry requires additional optics with respect to classical imaging, these measurements require much more photons to achieve a good signal-to-noise ratio for which the 3.6-m Devasthal Optical Telescope (DOT) facility is suitable.
\end{abstract}

\section{Introduction}

Some of the physical processes that produce or modify polarization in or around different types of astrophysical sources are: (i) Anisotropic scattering or reflection of continuum radiation; (ii) Anisotropic scattering of line radiation; (iii) Differential absorption or scattering by magnetically aligned non-spherical dust grains; (iv) Synchrotron radiation from charged particles in a magnetic field; (v) Zeeman effect or Paschen-Back effect in presence of a magnetic field; (vi) Stark effect (Electric field). Many properties of scattering dust clouds (e.g. the sizes, shapes and chemical composition of the scatterers, etc.) can be obtained through polarimetry. Even the content, structure and stratification of the atmosphere of some of the stars, planets or exoplanets can, in principle, be characterised without optically resolving them. Another important and unique capability of polarimetry is the measurement of magnetic fields. The High Accuracy Radial velocity Planet Searcher spectropolarimeter (HARPSpol) on the European Southern Observatory (ESO) 3.6-m telescope at La Silla (Chile; Snik et al. 2010), and the Echelle SpectroPolarimetric Device for the Observation of Stars (ESPaDOnS) on the Canada France Hawaii Telescope (CFHT) 3.6-m telescope (Hawaii; Donati 2003) are being used to probe the magnetic field structure in stars. NARVAL, a twin of ESPaDOnS, adapted to the specifics of the 2-m Telescope Bernard Lyot (TBL) at Pic du Midi (France) is fully dedicated to stellar spectropolarimetry. HARPSpol on ESO 3.6-m, ESPaDOnS on CFHT, and NARAVAL at TBL (Moutou et al. 2015) are also giving support to study the magnetic field structure of target sources of the BRITE nano-satellite constellation (Weiss et al. 2014). The addition of a polarimeter for continuum band or a spectropolarimeter will widen the science scope on the 3.6-m DOT. In this contribution, the basics of polarimetry and the scope citing a few problems that can be addressed with this 3.6-m telescope are discussed.

\footnotetext{
*email: joshi@prl.res.in
} 


\section{Description of light - Stokes' parameters}

Two orthogonal components of the electric vector of an electromagnetic wave of wavelength $\lambda$ moving in the direction $\mathbf{z}$ can be represented as:

$$
\begin{array}{lll}
E_{x}=E_{x 0} \cos \left(\omega t-\frac{2 \pi z}{\lambda}+\delta_{x}\right) & \text { or the real part of } & E_{x 0} e^{j\left(\omega t-\frac{2 \pi z}{\lambda}+\delta_{x}\right)} \\
E_{y}=E_{y 0} \cos \left(\omega t-\frac{2 \pi z}{\lambda}+\delta_{y}\right) & \text { or the real part of } & E_{y 0} e^{j\left(\omega t-\frac{2 \pi z}{\lambda}+\delta_{y}\right)}
\end{array}
$$

So, for a complete description of light, we need 4 parameters: the amplitude and phase of two orthogonal components of electromagnetic field, i.e. $E_{x 0}, E_{y 0}, \delta_{x}$, and $\delta_{y}$.

The set of 4 parameters $E_{x 0}, E_{y 0}, \delta_{x}$, and $\delta_{y}$ described by Eqs. (1) and (2) completely describe the nature of light, but this is not the most convenient set as they are related to the coordinate frame. The most convenient set is Stokes' parameters defined as:

$$
\begin{gathered}
I=\left(E_{x 0}^{2}+E_{y 0}^{2}\right), \\
Q=\left(E_{x 0}^{2}-E_{y 0}^{2}\right), \\
U=2 E_{x 0} E_{y 0} \cos \left(\delta_{x}-\delta_{y}\right) \\
V=2 E_{x 0} E_{y 0} \sin \left(\delta_{x}-\delta_{y}\right)
\end{gathered}
$$

The degree of linear polarization $P$ and the position angle $\theta$ of the polarization vector are expressed as:

$$
P=\sqrt{Q^{2}+U^{2}} / I \quad \text { and } \quad \tan 2 \theta=U / Q
$$

\section{Sources of error in astronomical polarimetry}

In modern polarimeters, the main source of error is related to photon noise, and other sources of error can be taken care off using fast modulation techniques. So here we discuss only photon noise. The photon flux follows Poisson statistics, and this is also true for the photo-electrons generated by the interaction of photons with the detector. The signal-to-noise ratio (SNR) depends on the number of detected photons: the higher the photon flux, the higher is the SNR. With $N$, the number of photons, $\sqrt{N}$ as photon noise, the the error in Stokes' parameters can be expressed as,

$$
\epsilon(Q / I)=\epsilon(U / I)=\sqrt{2 / N}
$$

Therefore, to reduce the error we need to: (i) count more photons; (ii) observe a wide range of wavelength simultaneously; (iii) use the detectors with the highest quantum efficiency in a particular spectral range; (iv) use of photon counting devices such as PMT, image tube.

\subsection{Error Estimate}

The number of photo-electrons produced per second by a photo cathode can be expressed as:

$$
N=3.95 \times 10^{18} \cdot \lambda \cdot \Delta \lambda \cdot F_{\lambda} \cdot T \cdot q \cdot D^{2} \cdot 10^{-0.4 m}
$$

where $F_{\lambda}$ is the flux density of a 0 th magnitude A0V star expressed in $\mathrm{W} / \mathrm{cm}^{2} . \mu \mathrm{m}, \lambda$ and $\Delta \lambda$ are the wavelength and the band width in $\mu \mathrm{m}$, respectively, $T$ is the total transmission including atmosphere and optics, $q$ is the detector quantum efficiency and $m$ is the star's magnitude. The constant $3.95 \times 10^{18}$ takes care of the units used here. Assuming $D=3.6 \mathrm{~m}, T=20 \%, \lambda=0.44 \mu \mathrm{m} \& \Delta \lambda=0.09 \mu \mathrm{m}$ (B-band of UBV system), $q=20 \%$ (a conservative number for presently available PMTs), $F_{\lambda}=7.2 \times 10^{-12} \mathrm{~W} / \mathrm{cm}^{2} . \mu \mathrm{m}$, and taking an integration time of $10 \mathrm{~min}$ on a $\mathrm{B}=17.5 \mathrm{mag}$ star with the 3.6-m DOT, the expected error is $\epsilon \sim 0.2 \%$. With this limit on the error, several interesting problems related to faint sources can be taken up on the 3.6-m DOT. For instance, 
BLLac objects show a reasonably high polarization ( 5-15\%), but the majority of sources are faint, so they make a good case for the 3.6-m DOT (more in Sect. 4). High-resolution spectropolarimetry, needed to study the magnetic field structure and chemical peculiarity of stars, can be taken up on the 3.6-m DOT. A few science cases of interest for us are discussed below.

\section{Histogram of BL Lac sources}

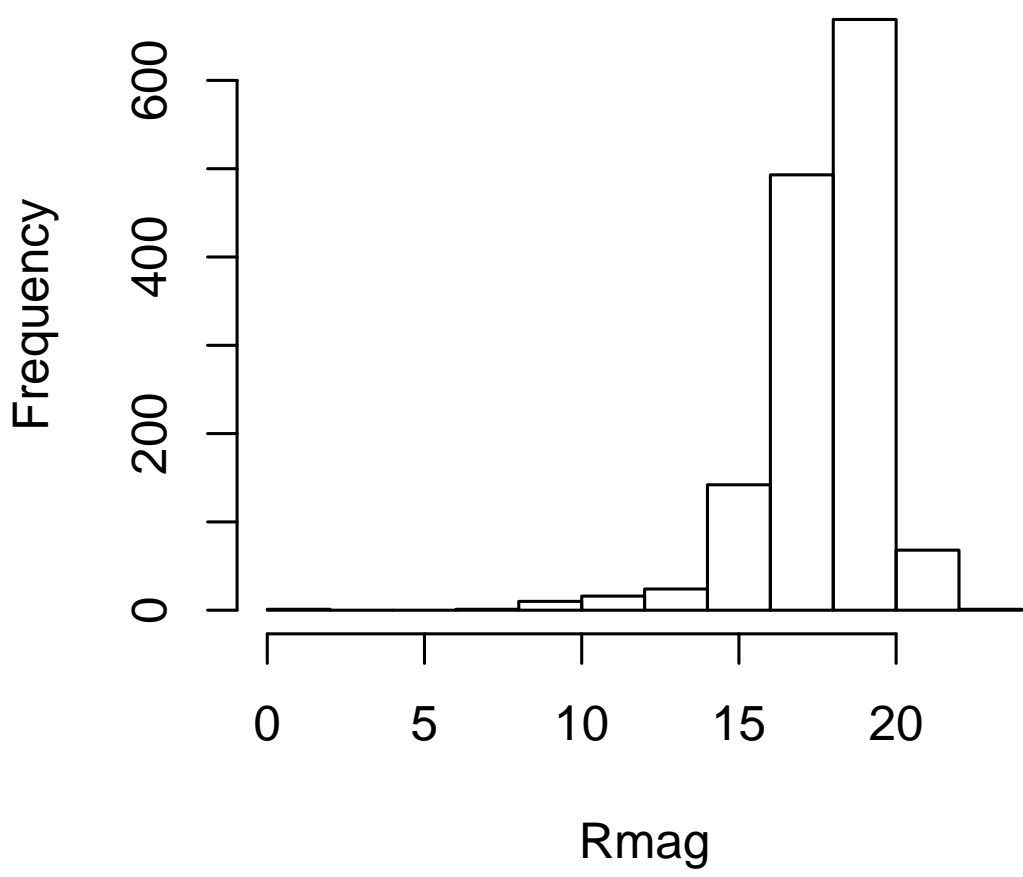

Figure 1: Histogram of BL Lac sources filtered from the Roma-BZCAT. Sources marked as BL Lac along with sources marked as "BL Lac - galaxy dominated" are considered.

\section{A few science cases}

Some problems which are of interest to us are listed here. Note that only a few problems are mentioned here.

1. BL Lac objects: The amplitude of the flux variation on short time scale is in general low $(<5 \%$ or so), and the same is true for the degree of polarization. To understand the inhomogeneities within the jet in blazars, high cadence polarization data with a high SNR are needed, which require a large telescope. These sources are highly polarised with emission mechanism being synchrotron process. Here we consider the BL Lac source OJ 287 for discussion purposes. OJ 287 shows an interesting flickering behaviour in the degree of polarization (Kulshrestha et al. 1984). Another interesting source is CGRaBS J0211+1051. This source was studied using observations on the 1.2-m telescope at Mt Abu observatory (Sunil et al. 2012). To improve the SNR, most of the observations were made in white light. On the 1.2-m Mt Abu telescope, BL Lac sources are being regularly monitored, but mostly in white light for better SNR. It would be very useful to observe such sources simultaneously in two filters, say B and R-bands, with high cadence and high SNR to confirm the shock-in-jet model (Marscher \& Gear 1985) which is believed to be responsible for such a flickering. Fig. shows the distribution of R-magnitude of 
BL Lac sources taken from the Multifrequency Catalogue of BLAZARS (Roma-BZCAT; Massaro et al. 2015). Most of the sources are within the 17-18 magnitude range. There are a good number of sources in the range 16-17 mag. For such sources, one can achieve a $0.1 \%$ polarimetric accuracy with the $3.6-\mathrm{m}$ DOT in the UBVRI bands in an integration time of $\sim 5 \mathrm{~min}$.

2. Polarimetry of solar system objects: The polarization behaviour of comets and asteroids at low phase angle $\left(<20^{\circ}\right)$ is not well understood. We studied a few comets at low phase angles (Joshi et al. 2010; Ganesh et al. 2009 and reference there in). In general comets (especially ecliptic comets) at small phase angles are at large distances, and hence they are faint. In addition to this we need to use narrow band filters to avoid emission bands. This further makes comet polarimetry at low phase angle difficult with small telescopes. The availability of the 3.6-m DOT will be helpful for this study.

3. Spectropolarimetry of chemically peculiar stars: The role of the magnetic field in chemically peculiar stars is of great interest. Knowledge of the magnetic field structure on chemically peculiar stars helps to understand the relationship between magnetic field geometry and the surface distribution of chemical elements (Russonarov et al. 2013). The addition of a high resolution spectropolarimeter as a back-end instrument on the 3.6-m DOT will greatly help research in this field.

In conclusion, there are several problems related to stellar physics, active galactic nuclei, solar system objects, etc. that can be studied using continuum bands or spectropolarimetry on the 3.6-m DOT.

\section{Acknowledgements}

This research work is supported by the Department of Space, Govt. of India. Local support to participate in first BINA workshop at ARIES, Nainital was provided by the BINA workshop organisers. We thank the referee for critical comments that improved this contribution.

\section{References}

Chandra S., Baliyan K. S., Ganesh S. J., Umesh C. 2012, ApJ, 746, 92

Donati, J.-F. 2003, ASPC, 307, 41

Ganesh S., Joshi U. C., Baliyan K. S. 2009 Icar, 201, 666

Joshi U. C., Ganesh S., Baliyan K. S. 2010, MNRAS, 402, 2744

Kulshrestha A. K., Joshi U. C., Deshpande M. R. 1984, Natur, 311, 733

Marscher A. P., Gear W. K. 1985, ApJ, 298, 114

Massaro E., Maselli, A., Leto C. et al. 2015, Ap\&SS, 357, 75

Mouillet, D., Aurière, M., Tbl, Narval Teams, 2006, in Proceedings of the Annual meeting of the French Society of Astronomy and Astrophysics Eds. D. Barret, F. Casoli, G. Lagache, A. Lecavelier, L. Pagani, 493

Rusomarov N., Kochukhov O., Piskunov N. et al. 2013, A\&A, 558, A8

Snik F., Kochukhov O., Piskunov N. et al. 2011. ASPC, 437, 237

Weiss W. W., Rucinski S. M., Moffat A. F. J. et al. 2014, PASP, 126, 573 